Check for updates

Cite this: RSC Adv., 2018, 8, 4072

Received 5th December 2017 Accepted 15th January 2018

DOI: $10.1039 / c 7 r a 13058 a$

rsc.li/rsc-advances

\title{
Flexible inorganic membranes used as a high thermal safety separator for the lithium-ion battery $\dagger$
}

\author{
Chuan Shi, (D) a Jianwei Zhu, ${ }^{\mathrm{b}}$ Xiu Shen, ${ }^{\mathrm{C}}$ Fuxing Chen, ${ }^{\mathrm{a}}$ Fanggang Ning, ${ }^{\text {a }}$ \\ Hongdi Zhang, ${ }^{b}$ Yun-Ze Long, (D) ${ }^{* a b}$ Xin Ning $^{\star a}$ and Jinbao Zhao (iD ${ }^{c}$
}

\begin{abstract}
A flexible $\mathrm{SiO}_{2}$ porous fiber membrane (SF) is prepared by electrospinning followed by calcination in this work. Compared with an organic substrate separator, the SF used as a separator will be an absolute guarantee of the battery thermal safety. The porosity of the SF is $88.6 \%$, which is more than twice that of a regular PP separator. Hydrophilic SF shows better electrolyte wetting ability and its high porosity enables the SF to absorb 633\% liquid electrolyte on average, while the lithium-ion conductivity reaches $1.53 \mathrm{mS} \mathrm{cm}^{-1}$. The linear sweep voltammogram testing of PP and SF suggested that SF, with great electrochemical stability, can meet the requirements of lithium-ion batteries. The cyclic and rate performances of batteries prepared with SF are improved significantly. Such advantages of the SF, together with its potential in mass production, make the SF a promising membrane for practical applications in secondary lithium-ion batteries.
\end{abstract}

\section{Introduction}

Lithium-ion batteries have been regarded as one of the major power sources for electric vehicles (EVs) and for the storage of new energy in a smart grid due to its high energy density and particularly stable cycle life., ${ }^{1,2}$ However, the potential safety hazard of the battery is exacerbated with the increase of capacity in current battery construction, in particular the use of separator materials. ${ }^{3-5}$ The separator is the core component of lithium-ion battery safety as it prevents the direct contact of the cathode and anode, while sustaining the free transport of the lithium-ions in the liquid electrolyte. ${ }^{6,7}$ Polyolefin porous membranes, particularly polyethylene (PE) and polypropylene (PP) separators became the most widely used products in the field of lithium batteries due to their high mechanical strengths, and excellent chemical and electrochemical stabilities. However, a safety issue, caused by the thermal shrinkage of the polyolefins at higher temperatures, has limited their further applications in energy storage systems, especially in hybrid electric vehicle (HEV) areas. ${ }^{\mathbf{8}, 9}$

To improve the thermal stability of the separator for the safety of lithium-ion batteries, the polyolefin membranes were modified $^{10-13}$ and lots of new type of separators ${ }^{14-18}$ were designed. Among them, the purely inorganic separators prepared with the

${ }^{a}$ Industrial Research Institute of Nonwovens \& Technical Textiles, College of Textiles \& Colthing, China

${ }^{b}$ Qingdao University, College of Physics Science, China

${ }^{c}$ Xiamen University, Department of Chemical Engineering, China

† Electronic supplementary information (ESI) available. See DOI: 10.1039/c7ra13058a sintering method could have the advantage of "absolutely" thermal stability, strong electrolyte absorption content and no dendrite puncturing problems. ${ }^{\mathbf{1 9 , 2 0}}$ However, their poor flexibility for cell winding assembly limits their practical application.

Flexible inorganic porous fiber membranes prepared with the electrospinning method were widely studied as insulation, selfcleaning and heat resistant filter. ${ }^{21-25}$ With high thermal, chemical, electrochemical stabilities and uniform micro-nano pores, they were expected to be a potential candidate as the separator for high safety batteries. In this work, polyvinyl alcohol (PVA) was used as template polymer and silicate (TEOS) was used as the source of $\mathrm{SiO}_{2}$, the PVA/TEOS precursor hybrid fiber membrane was prepared by the electrospinning method. The PVA was removed via high temperature sintering process and the basic inorganic microstructure of the precursor hybrid fiber membrane was retained, leading to flexible $\mathrm{SiO}_{2}$ inorganic porous fiber membrane (SF) materials. Such SF used as separator can guarantee the thermal safety of the batteries. The high porosity of SF obtained via the electrospinning technique allows it to absorb more liquid electrolyte, which further leads to high conductivities, thus improving the cyclic and rate performances of batteries. We believe that these advantages of the SF, together with potential in mass production, make the $\mathrm{SF}$ a promising candidate for practical applications in secondary lithium batteries.

\section{Experimental}

\section{Materials}

The starting materials included poly(vinyl alcohol) (PVA, $M_{\mathrm{w}}=$ 66 000, Aladdin Shanghai), phosphoric acid $\left(\mathrm{H}_{3} \mathrm{PO}_{4}\right.$; analytical 
grade, Aladdin Shanghai), tetraethyl orthosilicate (TEOS, Aladdin Shanghai) and deionized water. All materials were purchased commercially without further purification.

\section{Preparation of precursor solutions}

PVA solution was prepared at mass fraction $10 \%$ by dissolving it in the deionized water at $60{ }^{\circ} \mathrm{C}$ with vigorous stirring at high rate for $5 \mathrm{~h}$. Silica gel was prepared by hydrolysis and polycondensation by the dropwise addition of $\mathrm{H}_{3} \mathrm{PO}_{4}$ into TEOS, then stirring at room temperature for $4 \mathrm{~h}$. The mass ratio of TEOS : $\mathrm{H}_{2} \mathrm{O}: \mathrm{H}_{3} \mathrm{PO}_{4}$ is $1: 1: 0.02$. Then, $10 \mathrm{~g}$ PVA solution was dropped slowly into an equivalent weight of silica sol-gel at room temperature, then stirred for another $5 \mathrm{~h}$. Thus, the viscous precursor solution, used for the electrospinning, was obtained.

\section{Electrospinning and fabrication of SF}

The as-prepared gel was loaded into a syringe and the needle of the syringe was connected to a direct current (DC) high-voltage power source. The grounded stainless steel drum was placed $17 \mathrm{~cm}$ from the tip of the needle as the collector wrapped with aluminum foil and rotated at $40 \mathrm{rpm}$. The feeding rate of the precursor solutions by the syringe pump was $1 \mathrm{~mL} \mathrm{~h}^{-1}$. The fabrication chamber was kept at constant temperature $\left(25^{\circ} \mathrm{C}\right)$ and relative humidity (45\%). An electric potential of $18 \mathrm{KV}$ between the needle and the stainless steel drum was applied by the high-voltage power source and the hybrid nano-fibrous membranes were obtained on the aluminum foil. The hybrid nano-fibrous membranes were with heated at a temperature increase rate of $2{ }^{\circ} \mathrm{C} \mathrm{min}{ }^{-1}$ in the air until it reached $600{ }^{\circ} \mathrm{C}$ and maintained for $2 \mathrm{~h}$ to remove the organic constituents of PVA, the SF was obtained after being cooled down to room temperature.

\section{Electrode preparation and cell assembly}

Coin cells were prepared for battery performance tests. A mixture of slurry containing 5 wt $\%$ acetylene black (super-P), $5 \mathrm{wt} \%$ polyvinylidene fluoride (PVDF) and $90 \mathrm{wt} \% \mathrm{LiMn}_{2} \mathrm{O}_{4}$ (Qingdao Xinzheng Material Co., Ltd, China) in $N$-methyl pyrrolidine (NMP) was prepared for cathode of the cells. The PP separator (Nantong Tianfeng New Electronic Materials Co., Ltd.) and SF were used as separators for preparing the batteries. Batteries after injected by the equivalent weight of electrolyte $\left(1 \mathrm{~mol} \mathrm{~L}^{-1} \mathrm{LiPF}_{6}\right.$ dissolved in a mixed solution of dimethyl carbonate (DMC), ethylene carbonate (EC) and diethylcarbonate (DEC) with a volume ratio of $1: 1: 1$ ) were assembled in the argon gas within a glove box (Mbraun, Germany).

\section{Characterization of the separators}

The surface morphologies of pure PP separator and SF were investigated by (Phenom pro, Netherlands) at the acceleration voltage of $10 \mathrm{kV}$. Bruker AVANC III was used for the solid state NMR spectrometer test. Instron 3300 with a speed of 5 $\mathrm{mm} \min ^{-1}$ was used for the mechanical strength test. The porosity of the SF and PP separator can be calculated by the following equation with the $n$-butanol uptake method:

$$
P \%=M_{\mathrm{BuOH}} /\left(\rho_{\mathrm{BuOH}} \times\left(M_{\mathrm{BuOH}} / \rho_{\mathrm{BuOH}}+M_{\mathrm{m}} / \rho_{\mathrm{P}}\right)\right) \times 100 \%
$$

where $\rho_{\mathrm{BuOH}}$ and $\rho_{\mathrm{P}}$ represent the densities of $n$-butanol and polymer, while $M_{\mathrm{BuOH}}$ and $M_{\mathrm{m}}$ represent the $n$-butanol and mass of membrane, respectively.

A commercial drop shape analysis system (Powereach JC2000C1, Shanghai Zhongchen Digital Technique Equipment Co. Ltd., China) was used for the static contact angles text of PP separator and SF. The electrolyte uptake of the membranes was calculated by the following equation:

$$
\text { Uptake }(\%)=\left(W-W_{0}\right) / W_{0} \times 100 \%
$$

where $W_{0}$ and $W$ are the weights of the membranes before and after absorbing the liquid electrolyte, respectively. The linear sweep voltammograms (LSV) of Li/separator/stainless steel (SS) cell at a scan rate of $5 \mathrm{mV} \mathrm{s}^{-1}$ was measured in order to test the stability of PP and SF separator.

The ionic conductivities of PP separator and SF absorbing liquid electrolyte and sandwiched between two stainless steel electrodes were investigated by an electrochemical workstation (Solartron, SI-1260, England) with the frequency range of $1 \mathrm{kHz}$ to $100 \mathrm{kHz}$.

Cells with PP separator and SF were prepared to investigate the cyclic and rate performance using the electrochemical test equipment (LAND-V34, Land Electronic, China). To study the cyclic performances of the batteries, the cells were charged to $4.2 \mathrm{~V}$ and discharged to $3 \mathrm{~V}$ at $1.0 \mathrm{C}$ and the rate performance tests were carried out at current rates of $0.5 \mathrm{C}, 1.0 \mathrm{C}, 2.0 \mathrm{C}, 5.0 \mathrm{C}$, 10.0C and 0.5C.

\section{Results and discussion}

The SEM images of the precursor hybrid fiber membrane and the distributions of fiber diameter and pore size are shown in Fig. 1. It can be seen from the figure that the surface fibers of the

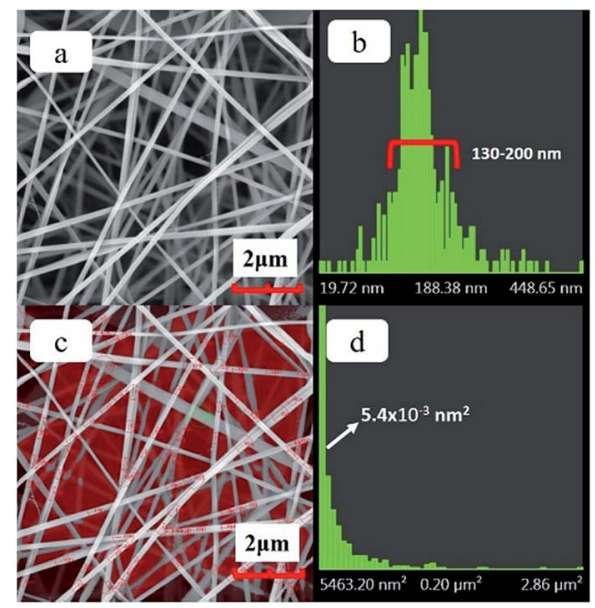

Fig. 1 (a) SEM image of the precursor hybrid fiber membranes, (b) the fiber diameter distribution chart of precursor hybrid fiber membranes, (c) the area of precursor hybrid fiber membrane pore size distribution statistics (d) the pore size distribution chart of precursor hybrid fiber membranes. 


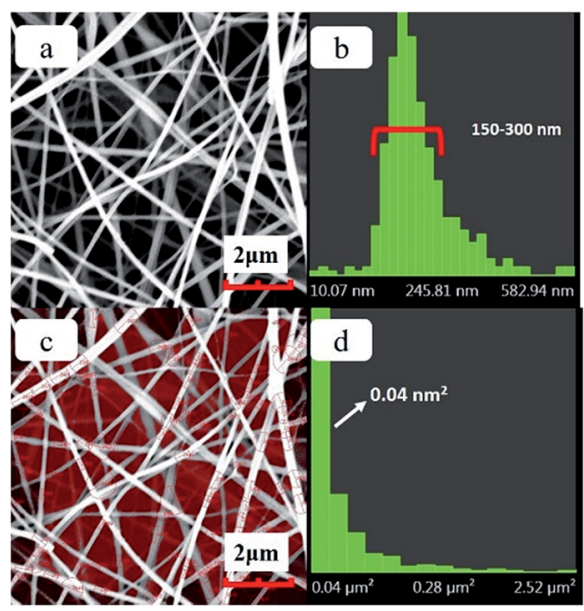

Fig. 2 (a) SEM image of the SF, (b) the fiber diameter distribution chart of SF, (c) the area of SF pore size distribution statistics (d) the pore size distribution chart of SF.

hybrid fiber membrane prepared by the electrospinning process is smooth and straight. The fiber diameter distribution chart (Fig. 1b) shows that the diameter of fibers has a concentrated distribution between a narrow range of $130-200 \mathrm{~nm}$, manifesting good quality of the fineness of fibers. The pore size of the hybrid fiber membrane is formed by the random layout of the fibers. After disregarding the incomplete pores at the edges of the image, the pore size distribution of hybrid fiber membranes is calculated (as shown in Fig. 1c and d). The pore size is concentrated at $0.05 \mu \mathrm{m}^{2}$, albeit this, there still exist pores with sizes larger than $3.00 \mu \mathrm{m}^{2}$.

The PVA component in the precursor hybrid fiber membrane was removed via high temperature sintering process to prepare the SF membrane. The SEM image of the SF and the distributions of the fiber diameter and pore size are shown in Fig. 2. It can be seen from Fig. 2a that the SF prepared by the sintering process retains the microstructure of the hybrid fiber membrane fairly well, with smooth fiber surface and uniform fiber fineness. Compared with the hybrid fiber membrane, fibers of SF are slightly curved. According to the fiber diameter chart (Fig. 2b), the diameters of the inorganic fibers are mainly between $150-300 \mathrm{~nm}$. The average diameter of the inorganic

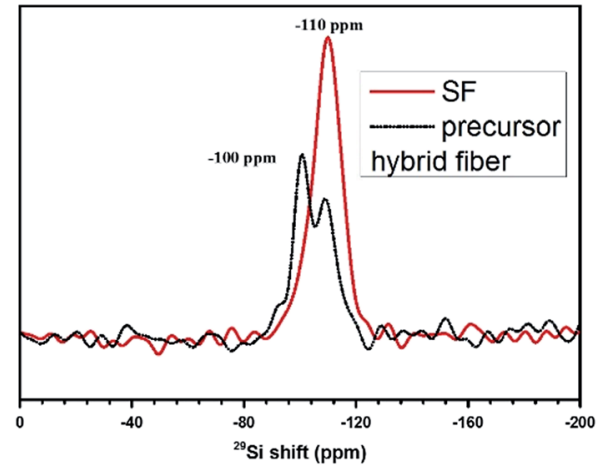

Fig. 3 The ${ }^{29} \mathrm{Si}$ NMR test of precursor hybrid fiber membrane and SF. fiber increased after the sintering process, suggesting that the fiber itself would undergo a physical changes such as nano-void formation during the sintering process, rather than a theoretical reduction in size due to the loss of the PVA composition in the fiber mass. Fig. $2 \mathrm{c}$ and d, also show that the pore size of the $\mathrm{SF}$ is concentrated at $0.04 \mu \mathrm{m}^{2}$, with more pores having a size between $0.04 \mu \mathrm{m}^{2}$ to $0.30 \mu \mathrm{m}^{2}$ and less pores having a size above $1.5 \mu \mathrm{m}^{2}$. The highly localized distribution of pore size indicate more uniformity in the pore sizes, which accompanies the physical changes of the fibers. Pore size uniformity is desirable and will contribute to the uniformity of the current in the lithium-ion battery and improves the performance of lithiumion battery. As shown in the Fig. S1, $\uparrow$ the pure PP separator shows an interconnected submicron porous structure which is a typical morphology from the dry process. In comparison, the pores of SF formed by the direct accumulation of fibers are large, which together with individual fiber characteristics, may be more conducive to absorbing liquid electrolyte and to the rapid conduction of $\mathrm{Li}^{+} .26,27$

The NMR of the SF and precursor hybrid fiber were shown in Fig. 3. It can be seen from Fig. 3, precursor hybrid fiber possess characteristic peaks of silicate species and silica. This shows that during the preparation of hybrid fiber membranes, TEOS has been partially hydrolyzed to silicic acid and silica, and the concentration of silicate is higher than that of silica. ${ }^{28}$ The NMR spectrum of SF was consistent with that of silica, indicating that TEOS completely hydrolyzed to $\mathrm{SiO}_{2}$ after calcined at high temperature, and finally the pure inorganic $\mathrm{SiO}_{2}$ nanofiber membrane was obtained. We also tested the mechanical strength of SF. The relevant data is in the Fig. S2. $\dagger$ The PP separator prepared with uniaxial stretching process will result in a significant mechanical strength decrease in this direction. Experiments show that even compared with the uniaxial stretching direction of the PP separator 7.5 MPa, the tensile strength of SF 3.5 MPa was still much lower. Obviously, the tensile strength of SF cannot fully meet the practical applications needs and we are currently also looking for ways to improve its mechanical strength.

Good separator material flexibility is critical in battery manufacturing, which requires flexibility for assembly processes in both pouch and cylindrical cell configurations. ${ }^{29} \mathrm{As}$ shown in Fig. 4, the SF is coiled around a glass rod to illustrate its good flexibility and can also recover the smooth morphology after winding.

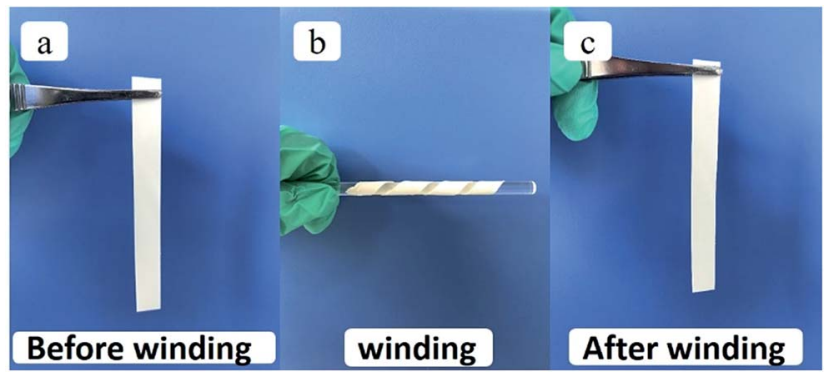

Fig. 4 Flexibility test of the SF separator before and after winding. 


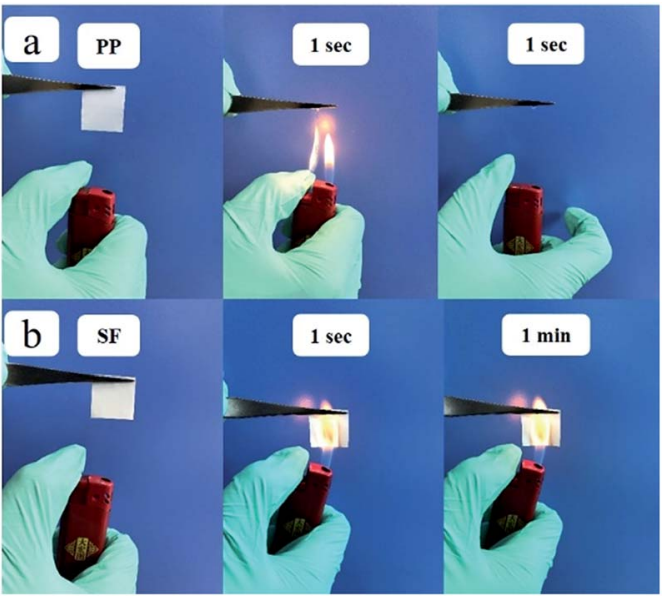

Fig. 5 The thermal stability test of the (a) PP at 1 second and (b) SF separator at 1 second and 1 minute.

The SF obtained after the high temperature sintering process could have the advantages of "absolute" thermal stability. To test the thermal stability of the separator under more severe conditions, the separators were ignited by a direct flame of a lighter and the results were shown in Fig. 5. The PP separator burned up immediately and was completely decomposed in just $1 \mathrm{~s}$. The morphologies and the flexibility of SF showed negligible changes in $1 \mathrm{~min}$. This flame-resistant properties of the SF are very useful for improving the thermal stability of lithium-ion batteries.

Separator and liquid electrolyte with good wettability can effectively sustain the electrolyte solution, and thus facilitate the lithium-ion transport between the cathode and the
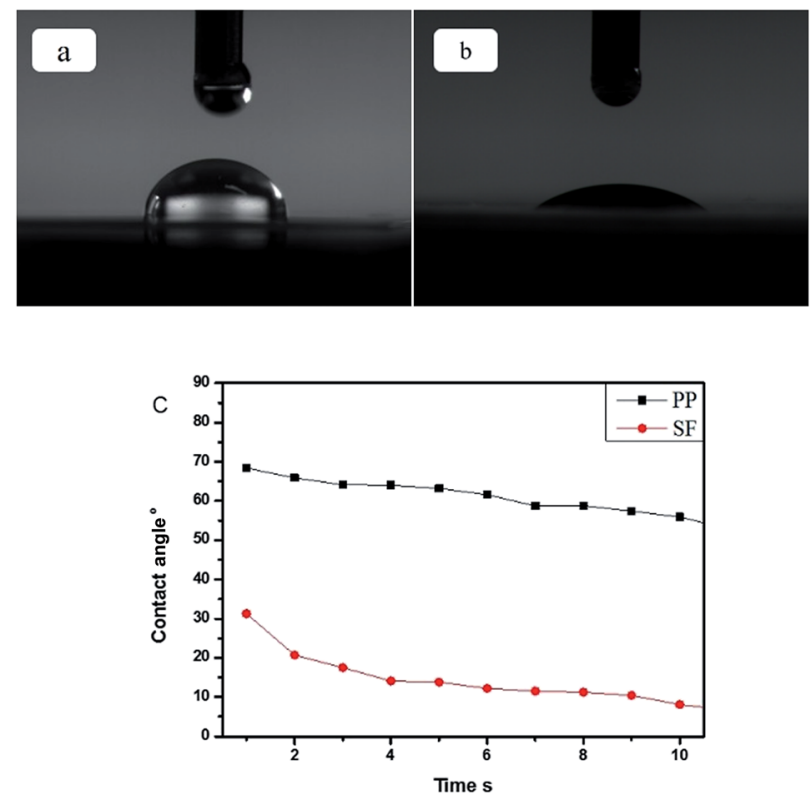

Fig. 6 (a) Contact angle for PP separator at $1 \mathrm{~s}$, (b) contact angle for SF separator at $1 \mathrm{~s}$, (c) contact angle of the separators changes with the time.
Table 1 The electrolyte uptake and ionic conductivity of the separators

$\begin{array}{lll}\text { Separator } & \text { PP separator } & \text { SF } \\ \text { Thickness } \mu \mathrm{m} & 40 & 45 \\ \text { Porosity\% } & 43.6 & 80.0 \pm 0.5 \\ \text { Average uptake\% } & 62.9 \pm 6 & 633 \pm 10 \\ \text { Ionic conductivity } \mathrm{mS} \mathrm{cm}^{-1} & 0.80 \pm 0.01 & 1.58 \pm 0.03\end{array}$

anode. ${ }^{30,31}$ Therefore, the wettability of the separator with the liquid electrolyte plays an important role in battery performance. The time dependence of the contact angle between the surface of the electrolyte and the separator surface reflects the wettability of the separator, which was measured and showed in Fig. 6. A high contact angle implies low wettability. As per the conventional/general procedure, a drop of the electrolyte was deposited on the separator surface, and the time-dependent contact angle was measured. As shown in Fig. 6, the contact angle for the PP separator was $70.0^{\circ}$ at $1 \mathrm{~s}$ and decreased to $55^{\circ}$ at $10 \mathrm{~s}$. In contrast, the SF has a contact angle as small as $32^{\circ}$ at $1 \mathrm{~s}$ and further decreased to below $10^{\circ}$ at $10 \mathrm{~s}$, indicating significant improvement in the wettability of the SF with the electrolyte.

A separator with high porosity and high liquid electrolyte uptake can retain more electrolyte and improve better ion transport. The porosity, electrolyte uptake and the ionic conductivity of PP and SF separators were measured and summarized in Table 1. The porosities of PP and SF are $43.6 \%$ and $88.6 \%$ respectively. The porosity of SF is nearly twice that of the PP. The electrolyte uptakes of PP and SF membranes are $62.9 \%$ and $633 \%$ respectively. The electrolyte uptake of SF is more than 10 times of the PP. SF having less weight and higher porosity results in a significant increase of the liquid electrolyte uptake. The ionic conductivities of PP separator and SF after soaking in the electrolyte solution are $0.80 \mathrm{mS} \mathrm{cm}^{-1}$ and 1.58 $\mathrm{mS} \mathrm{cm}{ }^{-1}$. The ionic conductivity of SF is nearly double the PP. The high porosity and high uptake of liquid electrolyte of SF is beneficial to increasing the ionic conductivity. High electrolyte uptake and ionic conductivity also help improve the cyclic and rate performance of lithium-ion batteries.

To achieve the practical application as lithium-ion battery separator, it is important to investigate the electrochemical

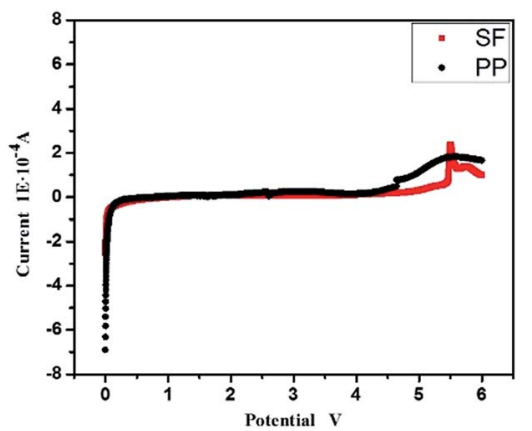

Fig. 7 The linear sweep voltammograms of PP and SF with Li and stainless steel electrode from $0 \mathrm{~V}$ to $6 \mathrm{~V}$. 


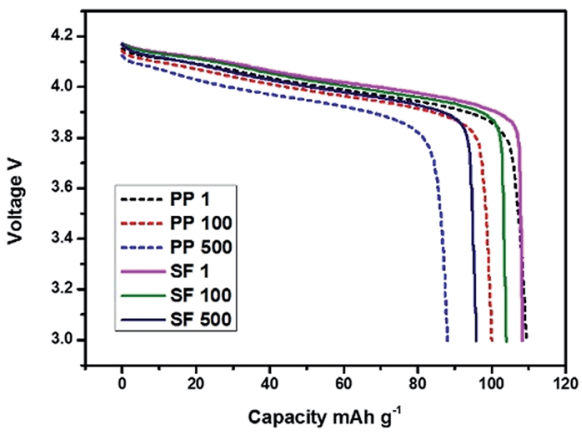

Fig. 8 The 1, 100, 500 cycles discharge curves of the batteries with PP separator and SF separator.

stability of the separator in the lithium-ion battery system. The linear sweep voltammograms (LSV) test of PP and SF with Li and stainless steel electrode were used to demonstrate the electrochemical stability of separator and Fig. 7 displayed the LSV result. PP separator shows obvious decomposition at about $4.8 \mathrm{~V}$. Compared with the PP separator, the SF separator shows no obvious decomposition below $5 \mathrm{~V}$, suggesting great electrochemical stability and it can meet the requirements of lithiumion battery.

Cells were charged and discharged for 500 cycles from 3.0 to $4.2 \mathrm{~V}$ at $1 \mathrm{C}$ rate for the cyclic performance test. Battery performances with PP and SF separators were shown in Fig. 8 and 9. As can be seen in Fig. 8, the traditional $\mathrm{LiMn}_{2} \mathrm{O}_{4}$ batteries discharge curve of PP and SF are almost the same, means there is no side effect for the use of SF membrane. Upon cycling, the $\mathrm{LiMn}_{2} \mathrm{O}_{4}$ cathode with PP separator and SF separator exhibited a high specific capacity of $110 \mathrm{~mA} \mathrm{~g}^{-1}$ at the first cycle and was stabilized above 500 cycles. The battery retained $87.5 \%$ capacity with SF separator and $80.5 \%$ with PP separator after 500 cycles. The improvement in cyclic performance of battery with SF separator was associated with the high electrolyte uptake and better wettability of the separator with the electrolyte.

Batteries were charged and discharged at the rates of $0.5 \mathrm{C}$, 1.0C, $2.0 \mathrm{C}, 5.0 \mathrm{C}, 10.0 \mathrm{C}$ and $0.5 \mathrm{C}$ for the rate performance test. As the Fig. 10 showed, the discharge capacities of batteries with PP separator and SF separator decreased gradually with the increase in rate. The capacity of the batteries displayed no big

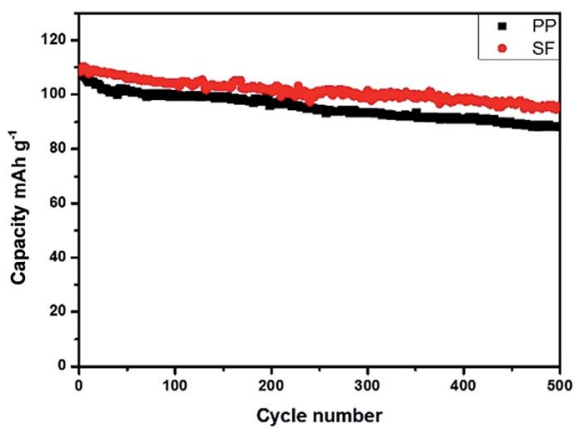

Fig. 9 The cyclic performance of the batteries with PP separator and SF separator.

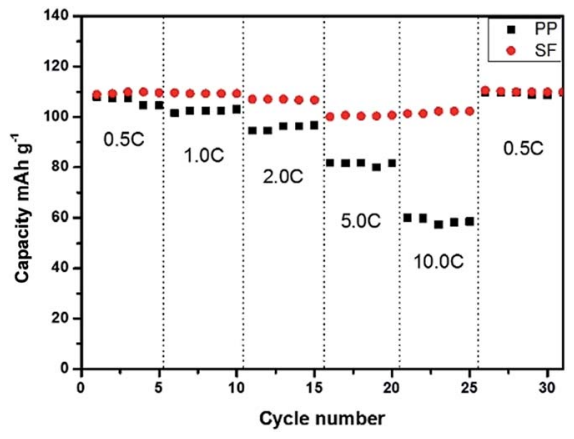

Fig. 10 The rate performance of the batteries with PP separator and SF separator.

difference in low rate and batteries prepared with SF showed higher rate capacity at high rate. The improvement in rate performance of battery was associated with the high electrolyte uptake and ionic conductivity of SF separator.

\section{Conclusions}

The SF can maintain the stability of the nano-porous structure even when it is ignited. The as-prepared SF used as separator can significantly enhance the thermal safety of the batteries which is critical for the development of electric vehicles and energy storage system. The porosity, electrolyte uptake and lithium-ion conductivity of the $\mathrm{SF}$ is $88.6 \%, 633 \%$ and 1.53 $\mathrm{mS} \mathrm{cm}{ }^{-1}$, which are much higher than those of the PP separator, resulting in improved cyclic and rate performances of batteries. Such functional advantages, together with the potential for mass production, makes SF suitable for practical applications in secondary lithium batteries.

\section{Conflicts of interest}

There are no conflicts to declare.

\section{Acknowledgements}

This work was supported by the National Natural Science Foundation of China (51373082 and 51673103), the Taishan Scholars Programme of Shandong Province, China (ts20120528), and the Shandong Provincial Key Research and Development Plan (2016GGX102011).

\section{References}

1 K. Brandt, Solid State Ionics, 1994, 69, 173-183.

2 G. Wu, H. Wu, K. Wang, C. Zheng, Y. Wang and A. Feng, RSC Adv., 2016, 6, 58069-58076.

3 S. I. Tobishima and J. I. Yamaki, J. Power Sources, 1999, 8182, 882-886.

4 J. Hassoun, S. Panero, P. Reale and B. Scrosati, Adv. Mater., 2009, 21, 4807-4810.

5 P. G. Balakrishnan, R. Ramesh and T. Prem Kumar, J. Power Sources, 2006, 155, 401-414. 
6 S. S. Zhang, J. Power Sources, 2007, 164, 351-364.

7 P. Arora and Z. Zhang, Chem. Rev., 2004, 104, 4419-4462.

8 D. Takemura, S. Aihara, K. Hamano, M. Kise, T. Nishimura, H. Urushibata and H. Yoshiyasu, J. Power Sources, 2005, 146, 779-783.

9 K. J. Kim, J.-H. Kim, M.-S. Park, H. K. Kwon, H. Kim and Y.-J. Kim, J. Power Sources, 2012, 198, 298-302.

10 C. Shi, P. Zhang, L. Chen, P. Yang and J. Zhao, J. Power Sources, 2014, 270, 547-553.

11 C. Shi, J. Dai, X. Shen, L. Peng, C. Li, X. Wang, P. Zhang and J. Zhao, J. Membr. Sci., 2016, 517, 91-99.

12 M.-H. Ryou, D. J. Lee, J.-N. Lee, Y. M. Lee, J.-K. Park and J. W. Choi, Adv. Energy Mater., 2012, 2, 645-650.

13 D. Fu, B. Luan, S. Argue, M. N. Bureau and I. J. Davidson, J. Power Sources, 2012, 206, 325-333.

14 X. Zhou, L. Yue, J. Zhang, Q. Kong, Z. Liu, J. Yao and G. Cui, J. Electrochem. Soc., 2013, 160, A1341-A1347.

15 J. Zhang, L. Yue, Q. Kong, Z. Liu, X. Zhou, C. Zhang, S. Pang, X. Wang, J. Yao and G. Cui, J. Electrochem. Soc., 2013, 160, A769-A774.

16 L. C. Zhang, Z. Hu, L. Wang, F. Teng, Y. Yu and C. H. Chen, Electrochim. Acta, 2013, 89, 310-316.

17 D. T. Wong, S. A. Mullin, V. S. Battaglia and N. P. Balsara, J. Membr. Sci., 2012, 394-395, 175-183.

18 J. Hao, G. Lei, Z. Li, L. Wu, Q. Xiao and L. Wang, J. Membr. Sci., 2013, 428, 11-16.
19 J. Chen, S. Wang, D. Cai and H. Wang, J. Membr. Sci., 2014, 449, 169-175.

20 H. Xiang, J. Chen, Z. Li and H. Wang, J. Power Sources, 2011, 196, 8651-8655.

21 H. Yu, J. Guo, S. Zhu, Y. Li, Q. Zhang and M. Zhu, Mater. Lett., 2012, 74, 247-249.

22 P. Milanović, M. Dimitrijević, R. Jančić Heinemann, J. Rogan, D. B. Stojanović, A. Kojović and R. Aleksić, Ceram. Int., 2013, 39, 2131-2134.

23 X. Mao, Y. Si, Y. Chen, L. Yang, F. Zhao, B. Ding and J. Yu, RSC Adv., 2012, 2, 12216.

24 Z. Ma, H. Ji, D. Tan, Y. Teng, G. Dong, J. Zhou, J. Qiu and M. Zhang, Colloids Surf., A, 2011, 387, 57-64.

25 Y. Si, X. Mao, H. Zheng, J. Yu and B. Ding, RSC Adv., 2015, 5, 6027-6032.

26 C. Shi, P. Zhang, S. Huang, X. He, P. Yang, D. Wu, D. Sun and J. Zhao, J. Power Sources, 2015, 298, 158-165.

27 W. Chen, Y. Liu, Y. Ma and W. Yang, J. Power Sources, 2015, 273, 1127-1135.

28 M. Lin, X. Shu, X. Wang, Q. Zhao, X. Han, L. Lin and X. Bao, Chin. J. Catal., 1999, 20.

29 K. Liu, W. Liu, Y. Qiu, B. Kong, Y. Sun, Z. Chen, D. Zhuo, D. Lin and Y. Cui, Sci. Adv., 2017, 3.

30 J.-Y. Sohn, J.-S. Im, J. Shin and Y.-C. Nho, J. Solid State Electrochem., 2011, 16, 551-556.

31 S.-J. Gwon, J.-H. Choi, J.-Y. Sohn, Y.-M. Lim, Y.-C. Nho and Y.-E. Ihm, J. Ind. Eng. Chem., 2009, 15, 748-751. 